\title{
Micro and Nano Structure of Electrochemically Etched Silicon Epitaxial Wafers ${ }^{\dagger}$
}

\author{
Ozren Gamulin, ${ }^{\mathrm{a}}$ Maja Balarin, ${ }^{\mathrm{a}}$ Mile Ivanda, ${ }^{\mathrm{b}, *}$ Marin Kosović, ${ }^{\mathrm{a}}$ Vedran Đerek, ${ }^{\mathrm{b}}$ Lara Mikac, ${ }^{\mathrm{b}}$ \\ Kristina Serec, ${ }^{a}$ Krešimir Furić, ${ }^{b}$ Davor Ristić, ${ }^{b}$ and Dubravka Krilov ${ }^{a}$ \\ ${ }^{a}$ University of Zagreb, School of Medicine, Department of Physics and Biophysics, Šalata 3b, 10 000, Zagreb, Croatia \\ ${ }^{\mathrm{b}}$ Ruđer Bošković Institute, Division of Materials Physics, Bijenička 54, 10000 Zagreb, Croatia
}

RECEIVED DECEMBER 21, 2011; ACCEPTED FEBRUARY 22, 2012

\begin{abstract}
Silicon epitaxial wafers, consisting of $280 \mu \mathrm{m}$ thick n-type substrate layer and 4-5 $\mu \mathrm{m}$ thick epitaxial layer, were electrochemically etched in hydrofluoric acid ethanol solution, to produce porous silicon samples. The resistivity of epitaxial layer was $1 \Omega \mathrm{cm}$, while the substrate was much better conductor with resistivity $0.015 \Omega \mathrm{cm}$. By varying the etching time, the micro- and nano-pores of different sizes were obtained within the epitaxial layer, and on the substrate surface. Due to the lateral etching the epitaxial layer was partially detached from the substrate and could be peeled off. The influence of etching time duration on the optical and structural properties of porous samples was investigated by Raman, infrared and photoluminescence spectroscopy. The samples were analysed immediately after the etching and six months later, while being stored in ambient air. The Raman spectra showed the shift in positions of transversal optical (TO) phonon bands, between freshly etched samples and the one stored in ambient air. Infrared spectra indicated the presence of $\mathrm{SiH}_{x}$ species in the freshly etched samples, and appearance of oxidation after prolonged storage. Photoluminescence spectra were very weak in freshly etched samples, but their intensity has increased substantially in six month period. (doi: 10.5562/cca1971)
\end{abstract}

Keywords: nano materials, silicon, electrochemistry, raman, infrared

\section{INTRODUCTION}

Since silicon processing technology is widely used, it would be advantageous to use silicon for opto-electronic devices, instead of the predominantly used III-V materials. Due to an indirect band gap, which results in a low radiative recombination rate, ${ }^{1}$ silicon is a poor light emitter at room temperature. Ever since, in 1990, Canham $^{2}$ discovered visible photoluminescence (PL) in porous silicon (PS), at room temperature, a large research effort has been made in this field as well as in the field of other silicon nanostructures. The origin of visible light emission from porous silicon is still a question of debate, but most researchers connect it with the structural properties of material. ${ }^{2,3}$

Standard method for PS preparation is electrochemical etching of bulk silicon in an ethanolic hydrofluoric acid (HF) solution. Anodization under galvanostatic conditions is the preferred approach for attaining wide range of porosities and thicknesses, because it supplies the required charge for the etching reaction at a constant rate. ${ }^{4,5}$ The most important quantity controlling the process is the current density, since it determines the rate of holes transport to the HF interface. ${ }^{6}$ Different models have been suggested to elucidate the mechanism of pore formation. All of them agree that for the electrochemical dissolution process holes are required, and that the process starts at arbitrary nucleation sites on the surface. Pores are formed and their walls are etched until the holes are depleted. ${ }^{7}$

Different models have been proposed to explain experimental data, and to connect structural properties of porous silicon with observed luminescence. The most accepted is quantum confinement model (QCM), according to which the strong visible emission is caused by the modification of band gap in silicon nanocrystals. ${ }^{2,3}$ Another model proposes that PL is extrinsic to silicon and originates from silicon complexes covering the surface of Si crystallites. ${ }^{8}$ There is also the hybrid model that incorporates features of both, the model of quantum confinement as well as the siliconbased compounds like $\mathrm{SiH}_{x}$, and $\mathrm{SiO}_{2}$ in PS. ${ }^{9}$

\footnotetext{
$\dagger$ Presented at the $34^{\text {th }}$ International Convention on Information and Communication Technology, Electronics and Microelectronics, May $23^{\text {rd }}-27^{\text {th }}, 2011$, Opatija, Croatia.

* Author to whom correspondence should be addressed. (E-mail: ivanda@irb.hr)
} 


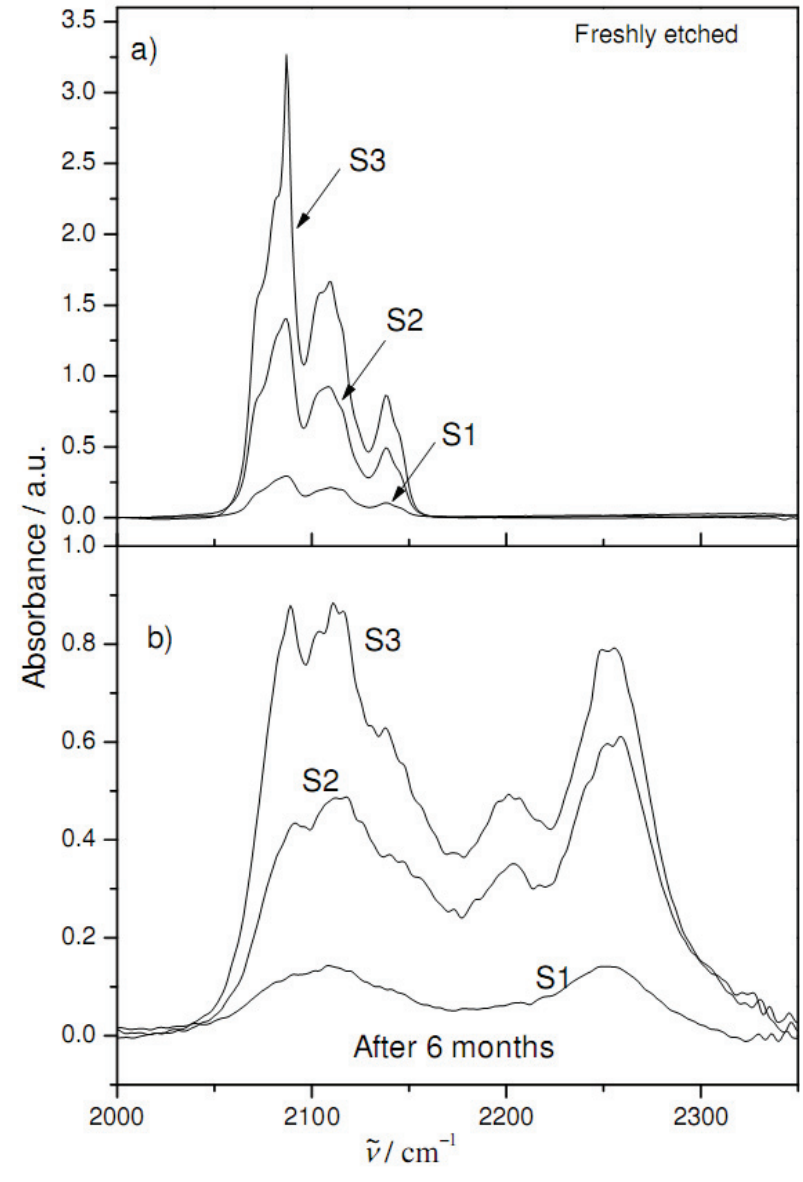

Figure 1. Infrared spectra of PS samples recorded after the etching process (a) and six months later (b).

In this paper, we present the results of Raman, PL and infrared measurements of PS samples prepared from low conductivity epitaxial layer grown on high conductivity substrate of n-type Si wafers. By varying the etching time, with the constant concentration of HF solution, pores of different sizes were obtained within the epitaxial layer and on the substrate surface. Freshly anodized samples showed small variation in the position of transversal optical (TO) phonon band in Raman spectra, and no PL spectra in the visible range. Storing samples for six month in ambient air caused numerous changes in acquired Raman and PL data. The peak position and the shape of TO phonon band in Raman spectra were changed and the PL emission spectra appeared.

\section{MATERIALS AND METHODS}

Commercially available silicon wafers, consisting of $280 \mu \mathrm{m}$ thick n-type (Sb doped) substrate and 4-5 $\mu \mathrm{m}$ thick $\mathrm{P}$ doped epitaxial layer, were electrochemically etched in a HF ethanol solution. The resistivity of the epitaxial layer was about $1 \Omega \mathrm{cm}$, while the resistivity of substrate was $0.015 \Omega \mathrm{cm}$. Aluminium foil was placed between the wafer and the base of the etching cell, to achieve better electrical contact. Three porous silicon samples, denoted S1, S2 and S3, were prepared by etching at a constant current density of $13 \mathrm{~mA} \mathrm{~cm}{ }^{-2}$, for 10 , 40 and 80 minutes, respectively. The etching process was performed under the fume hood illumination, with constant concentrations of $48 \% \mathrm{HF}$ in $96 \%$ ethanol (1:1 by volume).

After the etching process was completed, the $\mathrm{HF}$ ethanol solution was discarded from the etching cell. The samples were rinsed twice in ethanol and twice in pentane, and left to dry in air. The second pentane, added to the cell, was left there for $5 \mathrm{~min}$ to completely eliminate the capillary stress since pentane has a very low surface tension. ${ }^{5}$ The samples were investigated by Raman spectroscopy with two different excitation lines, photoluminescence (PL) spectroscopy and transmission FT infrared spectroscopy. Spectra were recorded from the top side of epitaxial layer and from the top surface of substrate layer. The Raman and PL spectra were recorded with Jobin Yvon T64000 triple spectrometer using a $514.5 \mathrm{~nm}$ excitation line of argon-ion laser. Both spectra were recorded in a single run at the same spot on the sample. The spot diameter was $6 \mu \mathrm{m}$ and the laser power was $20 \mathrm{~mW}$. The second excitation line used was in infrared spectral region $\lambda=1064 \mathrm{~nm}$ and the spectra ware recorded with FT-Raman module of Perkin-Elmer GX spectrometer. The infrared spectra were recorded with FT-IR module of the same instrument.

\section{RESULTS AND DISCUSSION}

The infrared spectra in the region between 2000 and $2400 \mathrm{~cm}^{-1}$ are presented in Figure 1. For freshly etched samples (Figure 1a) three vibrational bands, at approximately 2089,2110 and $2140 \mathrm{~cm}^{-1}$, can be distinguished. They were assigned to vibrations of $\mathrm{Si}-\mathrm{H}, \mathrm{Si}-$ $\mathrm{H}_{2}$ and $\mathrm{Si}-\mathrm{H}_{3}$ bonds, respectively. ${ }^{10,11}$ The intensity of these bands varies significantly with etching time. Sample S1, which was etched for the shortest time (10 min), shows very weak spectral features in this region. On the other hand the intensities of these bands for sample S3, which was etched for $80 \mathrm{~min}$, are much stronger. This indicates that longer etching time is generating more $\mathrm{Si}-\mathrm{H}_{x}$ bonds $(x=1,2$, and 3$)$. That can be connected with increasing number of pores and internal voids produced during the prolonged etching. When comparing the relative intensities of these three bands it is noticeable that they are changing with etching time too. The intensity of $\mathrm{Si}-\mathrm{H}$ band, at $\approx 2089 \mathrm{~cm}^{-1}$, is increased relative to the other bands, which suggests that, with increasing etching time, more $\mathrm{Si}-\mathrm{H}$ than $\mathrm{Si}-\mathrm{H}_{2}$ and $\mathrm{Si}-\mathrm{H}_{3}$ bonds were produced. The infrared spectra recorded 6 months later are presented in Figure 1b. Two additional vi- 


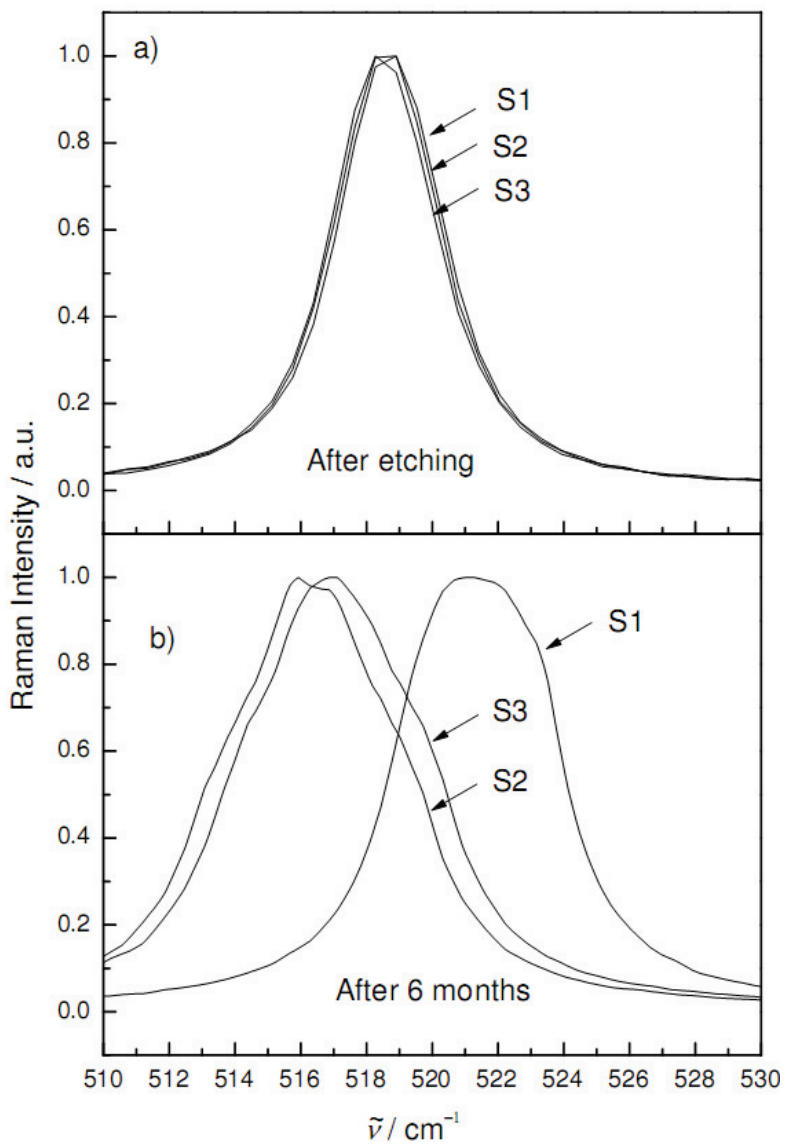

Figure 2. TO vibrational bands recorded, from the top side of epitaxial layer, after the etching process (a) and 6 months later (b).

brational bands, at 2200 and $2250 \mathrm{~cm}^{-1}$ appeared. At the same time the intensities of $\mathrm{Si}-\mathrm{H}_{x}$ vibrational bands are significantly reduced. Two new bands were assigned to stretching modes of $\mathrm{Si}-\mathrm{O}-\mathrm{Si}-\mathrm{H}$ species. These bonds were produced at the expense of $\mathrm{Si}-\mathrm{H}$ bonds during the process of oxidation. ${ }^{8,10,11}$ The relative intensities of three $\mathrm{Si}-\mathrm{H}_{x}$ vibrational bands were also changed. The ratio of band intensities at 2089 and $2110 \mathrm{~cm}^{-1}$ was changed in favour to the band at $2110 \mathrm{~cm}^{-1}$. This indicates that in oxidation process, where $\mathrm{Si}-\mathrm{O}-\mathrm{Si}-\mathrm{H}$ bond were created, more $\mathrm{Si}-\mathrm{H}$ then $\mathrm{Si}-\mathrm{H}_{2}$ and $\mathrm{Si}-\mathrm{H}_{3}$ bonds were used for the process of oxidation.

Figures 2 and 3 show Raman spectra of porous silicon samples recorded from the top side of epitaxial layer and from the top surface of substrate layer. It was shown in literature that transversal optical (TO) vibrational band at $520 \mathrm{~cm}^{-1}$ becomes broader and asymmetric and that its peak position shifts to lower wavenumber with decrease of nanocrystallite size. ${ }^{12}$ The Raman spectra recorded after the etching process, from the top side of the epitaxial layer (Figure 2a) and the top surface of the substrate (Figure 3a) show that they have etched out differently. This may be caused by the layered structure of used epitaxial wafers. Very thin epitax-

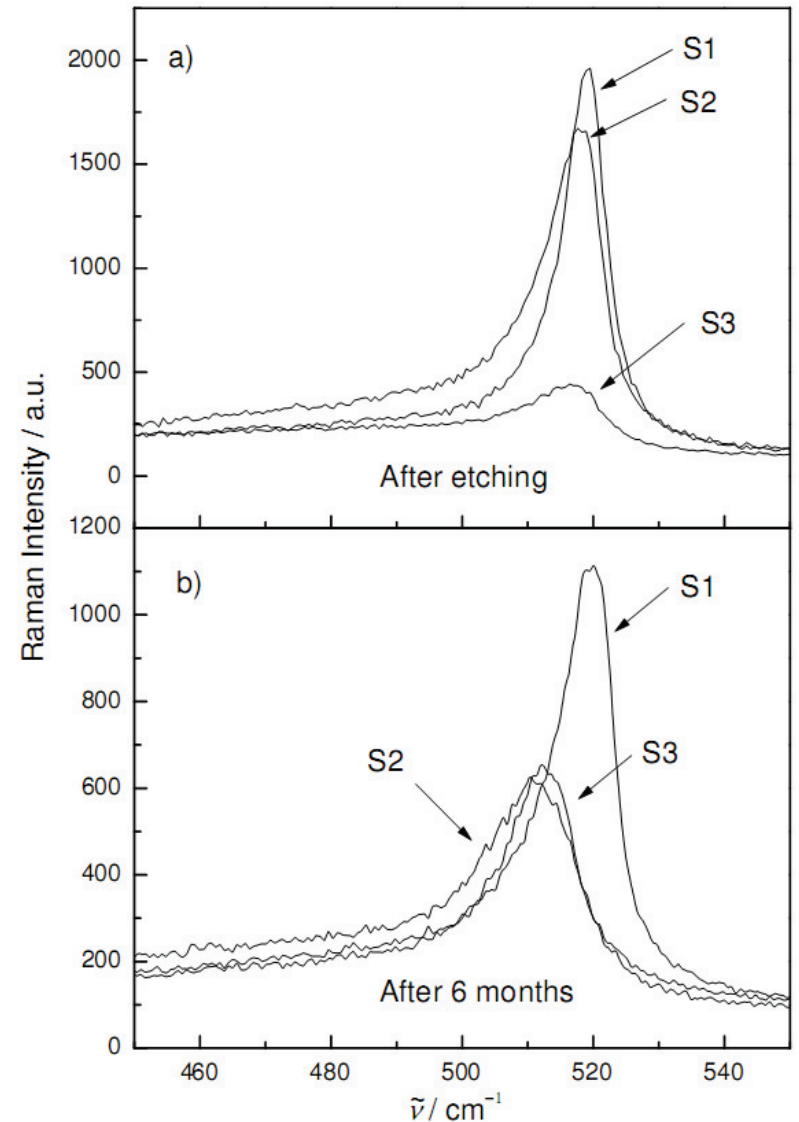

Figure 3. TO vibrational bands recorded, from the top surface of substrate, after the etching process (a) and 6 months later (b).

ial layer was etched through in probably less than five minutes, and pores had reached the interface between the epitaxial layer and the substrate. The etching process by dislocation is much faster when compared to the bulk silicon. For this reason the etching process of substrate layer is slower resulting in an increase of the lateral etching at the border between the epitaxial and the substrate layer, i.e. the substrate acts effectively as a barrier for penetration of F- ions. For this reason the epitaxial layer will be detached from the substrate by the lateral etching and hence it could be easily removed. At the same time, since resistivity of the epitaxial layer is almost hundred times higher than the resistivity of the substrate, there are much more available holes in the epitaxial layer than in the substrate. ${ }^{13}$ This may also promote the lateral etching and cause detachment of the epitaxial layer from the substrate. With further etching the porous structure is formed in the substrate.

When Raman spectra were recorded from the top side of epitaxial layer (Figure 2a), the peak position of TO vibrational band was fixed at $519 \mathrm{~cm}^{-1}$ for all three samples. Contrary to this when spectra were recorded from the top surface of substrate (Figure 3a), the peak position of TO vibrational band was shifted with an 


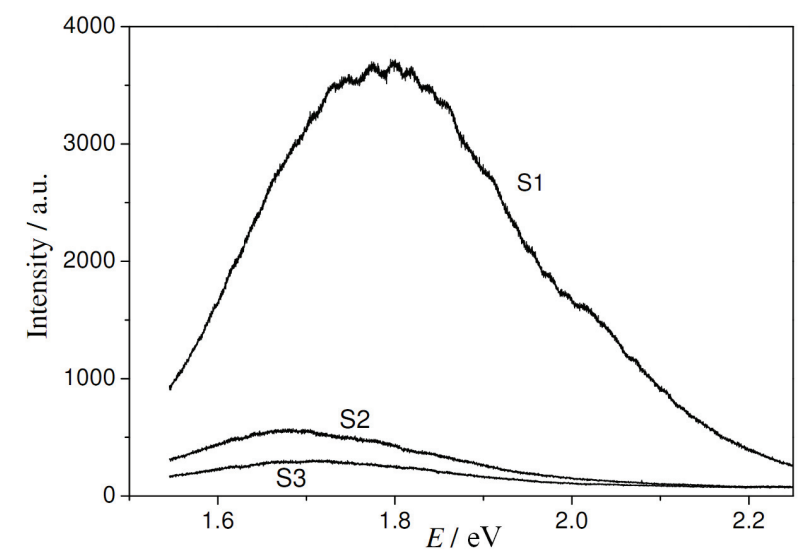

Figure 4. Photoluminescence spectra recorded from the top side of epitaxial layer 6 month after the etching.

increase in etching time. This may also suggest the existence of discontinuity in the etching process. It is known from literature that as the etching time increases the porosity increases too and hence the size of nanocrystallites decreases, ${ }^{5}$ which on the other hand shifts the peak position of the TO vibrational band to lower wavenumbers. ${ }^{14}$ This was observed in samples recorded from the top surface of substrate after the etching, which indicates that smaller nanostructures were formed with prolonged etching. On the other hand this was not observed in samples recorded after the etching from the top side of epitaxial layer. We can speculate that there exist two parallel processes which are pushing TO vibrational bands in opposite direction. The decrease in crystallite size would cause the shift of the TO peak position to lower wavenumbers. On the other hand, as the porosity of samples is increased, the compressive stress, caused by a lattice size discrepancy between porous and bulk silicon, is increased, too. ${ }^{15,16}$ The increase in the internal compressive stress would cause the shift in the peak position of the TO band towards the higher wave numbers. Consequently, it may compensate the effect of nanocrystallite size reduction. As a result of these two parallel processes the TO band position would remain approximately the same. The reason for strong influence of compressive stress on the peak position of TO vibrational band, in freshly etched samples, may be in different etching rate between the epitaxial and the substrate layer. According to literature, ${ }^{16}$ the stress in porous silicon is the strongest at the interface between the porous layer and the bulk silicon, and it is decreasing with distance from the interface. In these samples the etching process stops at the interface between two layers with different dopant concentration, and since the epitaxial layer was etched through and the etching of substrate has begun, the resulting porous silicon layer would be maximally 5-7 $\mu \mathrm{m}$ thick. This is larger from the penetration depth of the excitation laser which is $0.9 \mu \mathrm{m}$ for the $514.5 \mathrm{~nm}$ excita- tion. This indicates that the recorded Raman spectra are generated by the vibrations from the porous region with developed stress. That can, on the other hand, significantly influence the peak position of the TO vibrational band.

Figure $2 \mathrm{~b}$ and $3 \mathrm{~b}$ present TO vibrational bands in Raman spectra, recorded from the top side of epitaxial layer and the top surface of substrate, respectively, after the samples were being stored in ambient air for 6 months. When compared to the spectra recorded after the etching, where the peak position of the TO vibrational band was around $519 \mathrm{~cm}^{-1}$ (Figure 2a and 3a), it is noticeable that both the position and the shape of TO vibrational bands in all samples were changed. The peak position of the TO vibrational band of sample S1, which was etched for the shortest time $(10 \mathrm{~min})$, was shifted to $521 \mathrm{~cm}^{-1}$. At the same time the peak position of the TO bands of samples S2 and S3, which were etched for substantially longer time (40 and $80 \mathrm{~min}$ ), were shifted towards $516 \mathrm{~cm}^{-1}$. Since the change in the peak position of TO vibrational band can be attributed to the change in nanocrystallite size, ${ }^{17}$ and to the change of the induced stress in the porous silicon layer due to the oxidation of material, ${ }^{18}$ or to both, this may be an explanation for the observed oppositely directional shifts in samples etched in different time durations. Because of short etching time, the porosity of sample S1 was low, with small pore volume, so they behave like nanodefects. ${ }^{10,17}$ Prolonged etching time in samples S2 and S3 causes an increase in porosity, and development of nano and macro voids. From infrared spectra (Figure 1a), it is apparent that freshly etched sample S1 has the lowest concentration of $\mathrm{SiH}_{x}$ bonds. Their number was additionally decreased after 6 months of oxidation, with the generation of $\mathrm{Si}-\mathrm{O}-\mathrm{Si}-\mathrm{H}$ species (Figure $1 \mathrm{~b}$ ). Therefore, we assume that nanodefects in sample $\mathrm{S} 1$ were turned into $\mathrm{SiO}_{2}$, which is a standard compound generated during the oxidation of porous silicon. ${ }^{10}$ The nanodefects with $\mathrm{SiO}_{2}$ occupy much more volume then the nanodefects with $\mathrm{SiH}_{x}$ species or dangling bonds. Consequently, the compressive stress developed in the porous layer is increased, which causes the shift of TO vibrational band towards higher wavenumbers. Contrary to this in samples S2 and S3 the voids were saturated with $\mathrm{SiH}_{x}$ bonds, so oxygen will connect with existing hydrogen and produce $\mathrm{Si}-\mathrm{O}-\mathrm{Si}-\mathrm{H}$ bonds (Figure $1 \mathrm{~b}$ ). These bonds will attract electronic cloud from $\mathrm{Si}-\mathrm{Si}$ bonds towards oxygen atom, and as a consequence $\mathrm{Si}-\mathrm{Si}$ bonds are weakened, the stress in porous layer is reduced and the TO vibrational bands are shifted to lower wavenumbers.

The visible photoluminescence spectra recorded from the top side of epitaxial layer of oxidized samples (stored for 6 month in ambient air) are presented in Figure 4. At the same time the PL spectra from freshly etched samples were not observed. Since the infrared 
spectra (Figure 1a) show that freshly etched samples were not oxidized, and since it is known that in non oxidized porous silicon the threshold porosity is needed for efficient $\mathrm{PL}^{4}$ the absence of PL spectra leads to conclusion that all prepared samples have relatively low porosity. ${ }^{16}$ On the other hand it is also possible that, due to the discontinuity in the etching process, the concentration of nonradiative recombination centres, like dangling bonds, was high and that they quenched photoluminescence. After the oxidation, the dangling bonds might become saturated with oxygen which act as radiative recombination centres and increase the intensity of photoluminescence as especially is strong for the sample S1 (Figure 4). The photoluminescence in porous silicon is attributed to many different origins, ${ }^{10}$ from the quantum confinement effects and nanocrystal surface states connected to the specific defects. Hence it can be assumed that the structural defects, generated at the beginning of the etching process, were responsible for the observation of the photoluminescence. Those defects are passivated by $\mathrm{H}$ during the anodization, and hence freshly etched samples do not display PL. When number of $\mathrm{SiH}_{x}$ bonds was reduced (Figure 1b), due to the exposure of samples to ambient air, these defects may participate in the radiative recombination processes.

The strongest PL intensity was observed in sample $\mathrm{S} 1$, which was etched for the shortest time, which has the lowest amount of $\mathrm{SiH}_{x}$ and $\mathrm{Si}-\mathrm{O}-\mathrm{Si}-\mathrm{H}$ bonds and which has the lowest porosity. According to literature, ${ }^{4}$ the opposite behaviour would be expected, but on the other hand, in this paper the unusual samples, layered ntype silicon, were investigated, and that can influence the resulting porous material. In addition the position of the TO vibrational band in Raman spectra of oxidized samples (Figure 2b) indicates that the oxidation has introduced additional stress in the sample S1, which can also contribute to the appearance of efficient photoluminescence. ${ }^{21}$ Additional contribution to the efficient photoluminescence might be the formation of $\mathrm{SiO}_{2}$ bonds during the oxidation of sample S1, which increases the number of radiative recombination centres and hence results in the most intensive photoluminescence intensity (Figure 4).

The assumption of increased number of defects in the sample $\mathrm{S} 1$ is supported by the infrared photoluminescence spectra presented in Figure 5. Infrared PL band is another characteristic photoluminescence band of porous silicon ${ }^{17}$ covering the spectral region from 0.8 to $1.2 \mathrm{eV}$. Since the used instrument was FT Raman, only the part below $1.1 \mathrm{eV}$ has physical meaning. Shoulders above that energy are attributed to artefacts connected with instrument configuration. ${ }^{19}$ The literature $^{17}$ indicates that in p-type silicon material the maximum of infrared PL band can be shifted with the porosi-

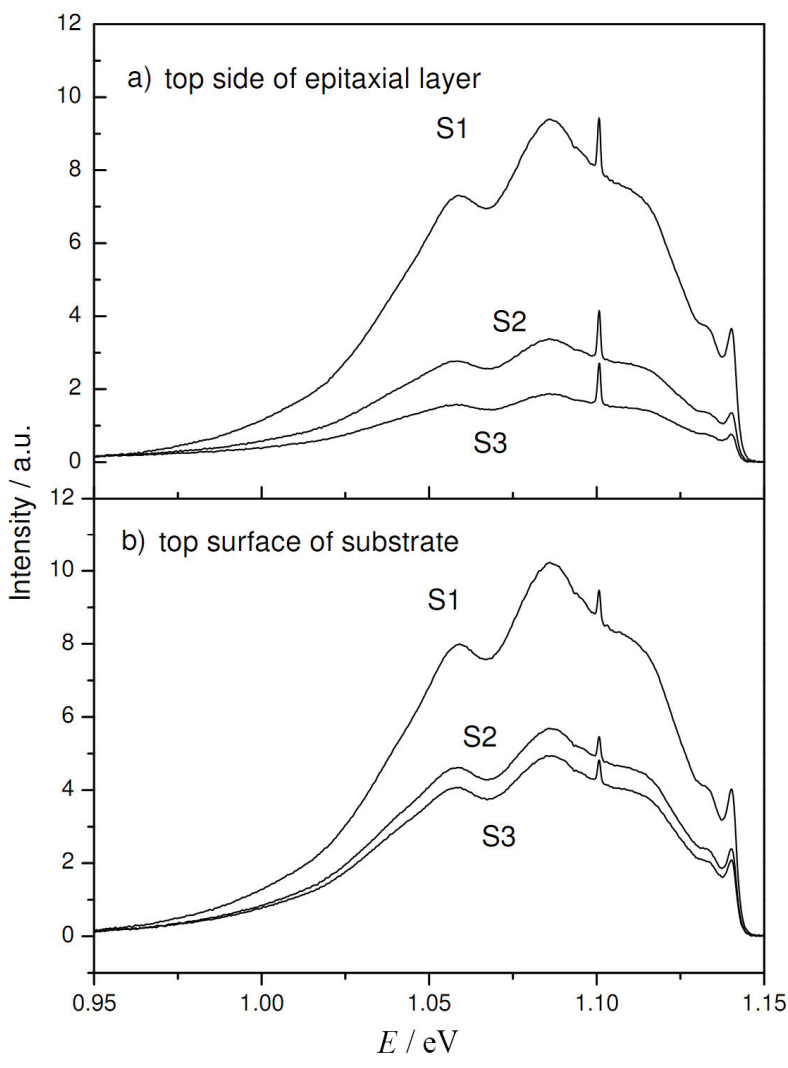

Figure 5. IR luminescence spectra recorded from the top side of epitaxial layer (a) and from the top surface of substrate (b).

ty, but that in the case of n-type semiconductor the position of the PL peak is fixed. The origin of the IR photoluminescence band is not completely understood, but several authors connected it with the point defects in porous material. ${ }^{10,17,18,20}$ Figures $5 \mathrm{a}$ and $5 \mathrm{~b}$ present the infrared photoluminescence spectra recorded, from the topside of epitaxial layer and from the top surface of substrate respectively, from oxidized samples stored in air for six month. Both images show that the most intensive PL is exhibited by the sample $\mathrm{S} 1$ which supports the assumption that its intensive photoluminescence is caused by the defects generated during oxidation process.

\section{CONCLUSION}

We investigated porous silicon produced, from n-type silicon wafers with differently doped substrate and thin epitaxial layer, in different etching time duration. The discontinuity of the etching process was observed at the interface between the epitaxial layer and the substrate. As a result two porous layers with different structural and photoluminescence properties were produced. The fixed position of the TO Raman vibrational band, in freshly etched porous silicon, was connected with the 
influence of compressive stress caused by the lattice dimension discrepancy. The blue shift of the TO vibrational band peak position, observed in S1 sample after oxidation, is explained with the increase of stress due to the generation of $\mathrm{SiO}_{2}$ in samples nanovoids. During the oxidation of $\mathrm{S} 2$ and $\mathrm{S} 3$ samples $\mathrm{Si}-\mathrm{O}-\mathrm{Si}-\mathrm{H}$ bonds were generated. Oxygen in these bonds attracts an electron cloud from $\mathrm{Si}-\mathrm{Si}$ bonds inside nanocrstals, and consequently weakens these bonds. Therefore, the position of TO band was shifted to lower wave numbers. The photoluminescence measurements showed that no PL spectra exist without the oxidation of the porous silicon, regardless of etching time. After the oxidation, the number of nonradiative recombination canters was reduced and defects which act as radiative centres were produced causing the appearance of PL. Increased number of defects was confirmed with the infrared photoluminescence spectra.

\section{REFERENCES}

1. P. Y. Yu and M. Cardona, Fundamentals of Semiconductors, Springer, Heidelberg, 2010.

2. L. T. Canham, Appl. Phys. Lett. 57 (1990) 1046-1048.

3. V. Lehmann and U. Gösele, Appl. Phys. Lett. 58 (1991) 856-858.

4. A. G. Cullis, L. T. Canham, and P. D. J. Calcott, J. Appl. Phys. 82 (1997) 909-965.

5. Z. Gaburro, N. Daldosso, and L. Pavesi, Porous Silicon, in: F. Bassani, J. Liedl, P. Wyder (Eds.), Encyclopedia of Condensed Matter Physics, Elsevier Ltd, 2005, pp. 391-401.

6. W. Theiß, Surf. Sci. Rep. 29 (1997) 91-192.
7. B. Lillis, C. Jungk, D. Iacoppino, A. Whelton, E. Hurley, M. M. Sheehan, A. Splinter, A. Quinn, G. Redmond, W. A. Lane, A. Mathewson, and H. Barney, Biosens. Bioelectron. 21 (2005).

8. K. Goda, R. Tanaka, Y. Honda, K. Inoue, and H. Ohno, Mat. Res. Soc. Symp. Proc. 638 (2001).

9. A. Roy, K. Jayararn, and A. K. Sood, Solid State Commun. 89 (1994) 229-233.

10. O. Bisi and S. Ossicini, L. Pavesi, Surf. Sci. Rep. 38 (2000) 1-126.

11. Y. H. Ogata, T. Tsuboi, T. Sakka, and S. Naito, J. Porous Mater. 7 (2000) 63-66.

12. A. K. Arora, M. Rajalakshim, and T. R. Ravindran, Phonon Confinement in Nanostructured Materials, in: H. S. Nalwa (Ed.) Encyclopedia of Nanoscience and Nanotechnology, American Scientific Publishers, 8, 2004, pp. 499-512.

13. R. A. Smith, Semiconductors, Cambridge University Press, Cambridge, 1978.

14. I. Gregora, B. Champagnon, and A. Halimaoui, J. Appl. Phys. 75 (1994) 3034-3039.

15. R. J. Martin-Palma, L. Pascual, A. Landa, P. Herrero, J. M. Martinez-Duart, Appl. Phys. Lett. 85 (2004) 2517-2519.

16. S. Montas, F. Agullo-Rueda, J. D. Moreno, F. Ben-Hander, and J. M. Martinez-Duart, Thin Solid Films 401 (2001) 306-309 .

17. L. T. Canham, Properties of Porous Silicon, INSPEC, London, 1997.

18. S. Manotas, F. Agullio-Rueda, J. D. Moreno, F. Ben-Hander, R. Guerrero-Lemus, and J. M. Martinez-Duart, Phys. Stat. Sol. (a) 182 (2000) 245-248.

19. J. D. Webb, D. J. Dunlavy, T. Ciszek, R. K. Ahrenkiel, M. W. Wanlass, R. Noufi, and S. M. Vernon, App. Spec. 47 (11) (1993) 1814-1819

20. A. Perez-Rodriguez, A. Cornet, J. R. Morante, J. Jimenez, P. L. F. Hemment, K. P. Homewood, J. Appl. Phys. 70 (1991) 16781682 .

21. Y. M. Huang, F.-F. Zhou, B.-G. Zhai, Proceedings of SPIE-The International Society for Optical Engineering, 2009, pp. 7375. 